
\title{
A TANDEM MASS-SPECTROMETRIC METHOD OF COSMOGENIC ISOTOPE
ANALYSIS
}

\author{
A. K. PAVLOV, V. T. KOGAN
}

A. F. Ioffe Physico-Technical Institute, Academy of Sciences, Polytechnicheskaya 26

St. Petersburg 194021 Russia

and

\section{G. Y. GLADKOV}

St. Petersburg State Technical University, Polytechnicheskaya 29, St. Petersburg 195251 Russia

\begin{abstract}
We propose an original method for analysis of low-concentrations of stable and long-lived radioactive nuclides. We discuss the parameters of the main features of the "Trace" spectrometer (a multicharged-ion laser source, a highly sensitive time-of-flight mass spectrometer, a charge-exchange chamber and a mass spectrometer for positive and negative single-charged ion analysis). We also compare these features with conventional AMS devices.
\end{abstract}

\section{INTRODUCTION}

We describe here the setup of our Tandem mass spectrometer, "Trace," which was designed in the Leningrad Physico-Technical Institute and is now under construction. Kocharov et al. (1990) discussed the basic operating principles at the Fifth Accelerator Mass Spectrometry (AMS) Conference in Paris. Figure 1 shows the principal layout of the setup, which includes a multicharged ion laser source combined with a highly sensitive time-of-flight (TOF) mass spectrometer and a charge-exchange chamber for the interaction of the ions with energy of about $1 \mathrm{keV} /$ nucleon passing through thin foils or gases. The mass spectrometer can be used for positive and negative single-charged ion analysis. An accelerator of any type can be used as an exit device of the Trace spectrometer for isobar discrimination and noise suppression.

\section{Methodology}

The basic mass-spectrometric problem is the separation of molecular ions with the same masses and isobars from the ions of interest. We resolve this by using an original method based on ideas from AMS.

In the first step, the high-efficiency laser ion source produces the ions with charge $Q>+2$, which are then separated by a TOF mass spectrometer. Because molecular ions with $Q>+2$ are unstable, they are eliminated by the first mass-spectrometer element of our setup. This mass spectrometer is also the separator from the main isotope.

The proportion of the ions in the appropriate charge state produced by the ion source can reach $50 \%$. The total current in these experiments was $10^{-7}-10^{-6} \mathrm{~A}$, and can be increased easily by using a more powerful laser.

The laser source is expected to have a lower background than the standard cesium sputtering source for AMS. This feature is a significant advantage for stable or long-lived radioactive nuclide measurements. We know that the laser source produces ion beams with a very wide spread in angle and energy distribution. Thus, we have designed a new mass spectrometer based on a combination of TOF separation and space focusing by magnetic field. The mass spectrometer shown in Figure 2 can analyze the ions with a wide spread in energy and angle distribution: solid angle - up to 1 sterad; energy - 10-160 eV. 
272 A. K. Pavlov, V. T. Kogan and G. Y. Gladkov

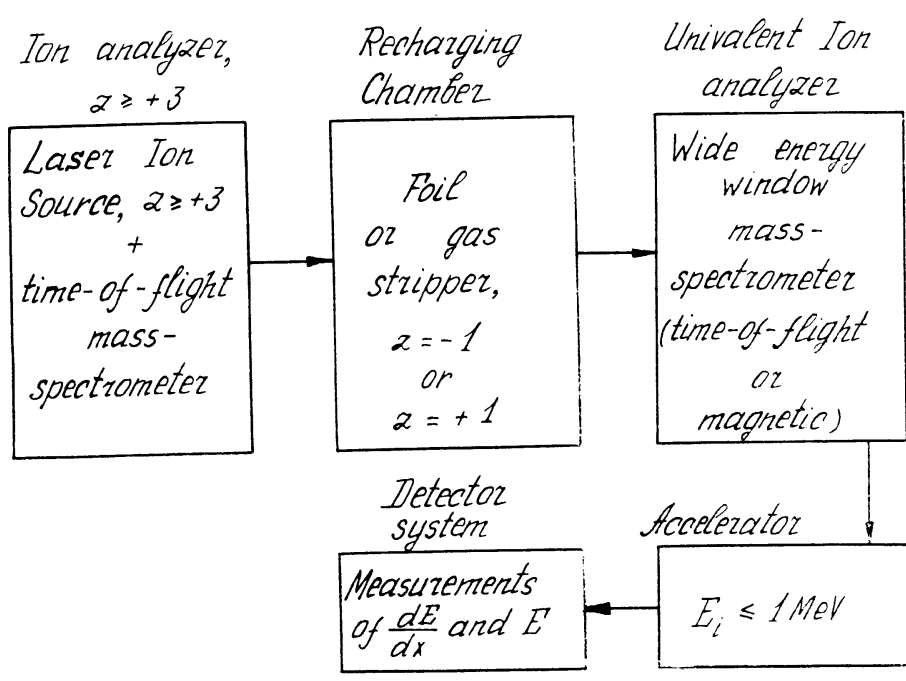

Fig. 1. Block diagram of the Trace setup

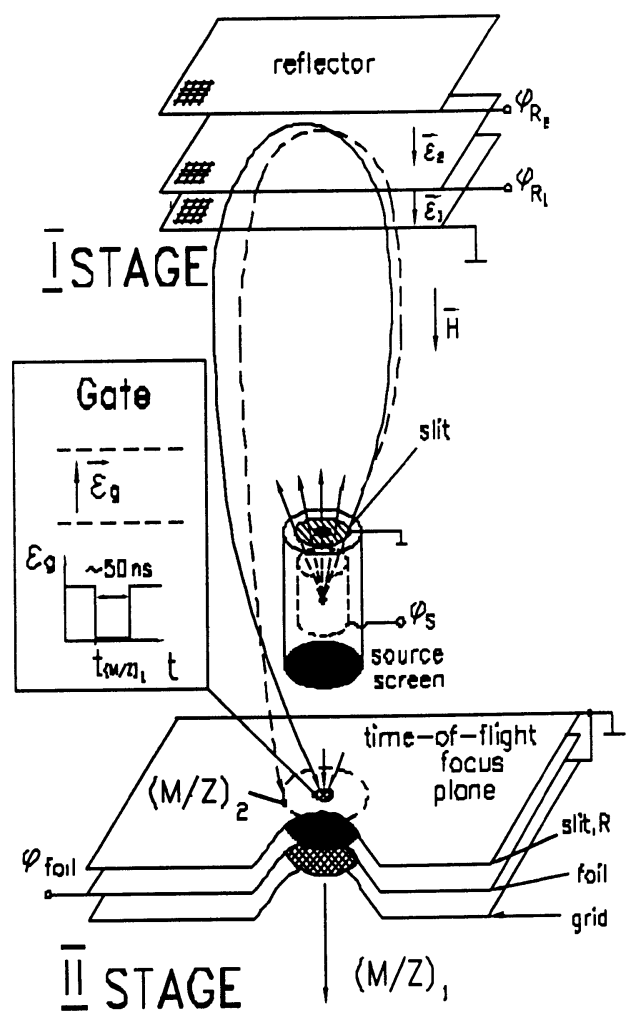

Fig. 2. Magnetic time-of-flight mass spectrometer

T.O.F. MASS-SPECTROMETER 
In the first step, ions are accelerated up to $U=1 \mathrm{kV}$, and pass through the first drift region. Then they are reflected by an electrostatic mirror (Smikk \& Dubensky 1984) and pass through the
second drift region. The total TOF, $t$, is described as

$$
\begin{gathered}
\mathrm{t}_{\mathrm{f}}(\mathrm{T})=(\mathrm{M} / 2 \mathrm{z})^{0.5} \cdot\left(\mathrm{L} / \mathrm{T}^{0.5}+4 \cdot \mathrm{T}^{0.5} / \mathrm{E}\right) \\
\mathrm{T}=\mathrm{ezU}+\mathrm{t}_{0}
\end{gathered}
$$

where $\mathrm{L}=$ drift length; $\mathrm{T}_{0}=$ initial ion kinetic energy; $\mathrm{E}=$ electric field intensity. This allows us to obtain TOF focusing of ions with different energy on the same plane, for any kind of ion. The mirror consists of two parts for better focusing.

The TOF system is placed in a uniform magnetic field, $\mathrm{H}$, parallel to the electric field in the reflector. Thus, certain mass ions with very wide angles are collected to focus at a point where $t_{r}$ $=t_{f}$, and $t_{r}=(M / z)(c / H)$. Other ions are collected in concentric rings of radius, $R$, on the same plane. An electrostatic gate is placed at the focal point to pass ions under investigation into the recharging chamber.

\section{RESULTS AND DISCUSSION}

Figures 3 and 4 show the results of our calculations of mass-spectrometric parameters. In the example, the energy window was $150 \mathrm{eV}$ and the TOF mass resolution was equal to 600. Figure 4 shows the Monte-Carlo calculation results of scattered ion background. Screens installed in strategic parts of the mass spectrometer can decrease scattering, because scattered ions can hit the thin foil only from a few regions of the electrostatic mirror.

In the next step, ions with energy of about $1 \mathrm{keV} /$ nucleon pass through the thin foil. Ions with positive charge, $\mathrm{Q}>+2$ are recharged; their charge-state distribution after the foil depends on their velocity and the foil chemical composition only. For the ion velocity, $V=10^{7} \mathrm{~cm} / \mathrm{s}$, the efficiency

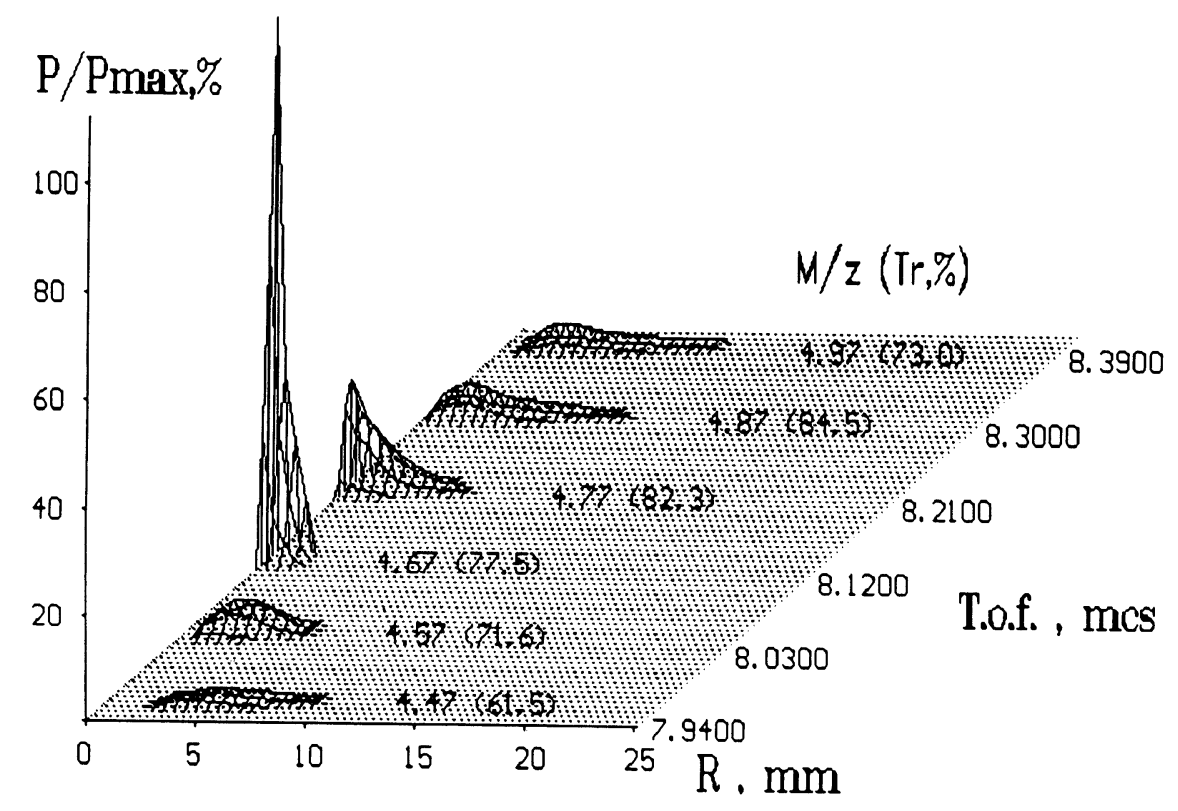

Fig. 3. Transmission and mass resolution of ion beams 


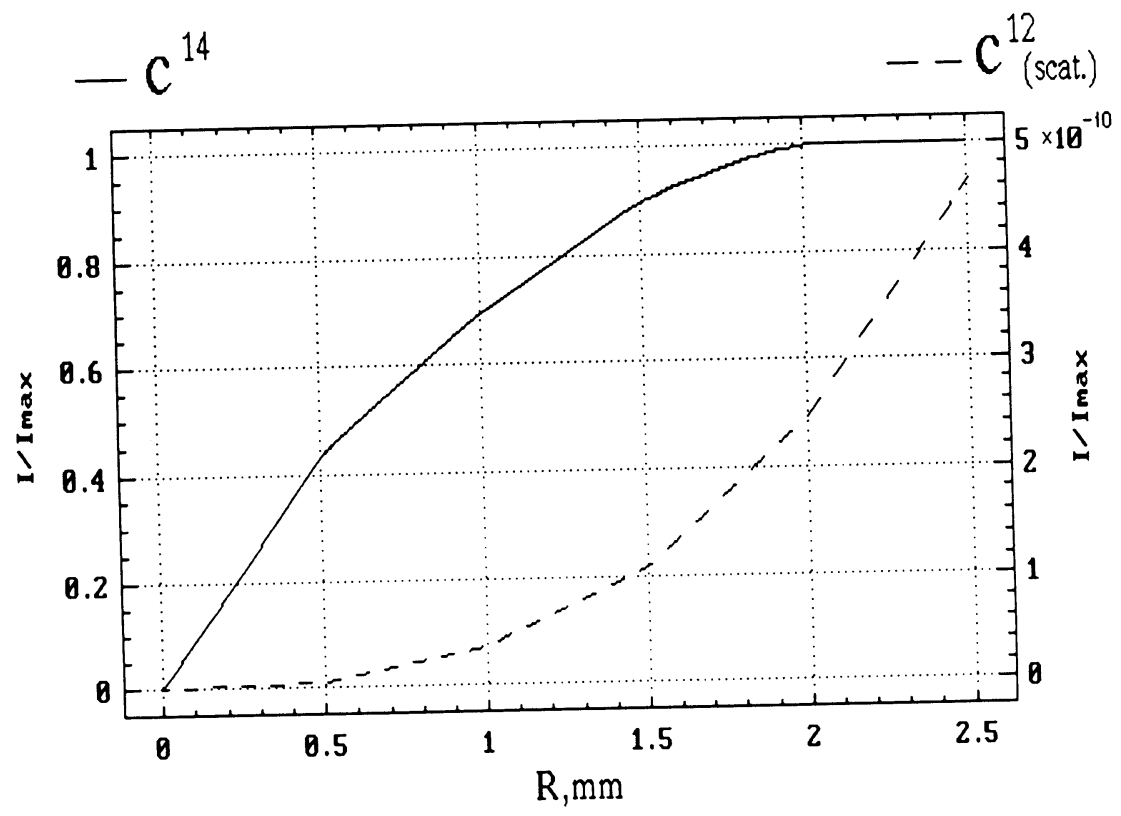

Fig. 4. Scattering ions intensity on the surface of thin foil

of the negative ion production can be from $1 \%$ to $10 \%$ for ${ }^{14} \mathrm{C},{ }^{26} \mathrm{Al},{ }^{36} \mathrm{Cl},{ }^{129} \mathrm{I}$, etc. (Fig. 5) (Shima, Mikumo \& Tawara 1986). For some isotopes, stable negative ions of their isobars do not exist (e.g., ${ }^{14} \mathrm{~N}-{ }^{14} \mathrm{C},{ }^{26} \mathrm{Mg}-{ }^{26} \mathrm{Al}$ pairs). Thus, the subsequent mass spectrometric stage should totally exclude these isobars.

Another method can be used if foil does not efficiently produce stable negative ions $\left({ }^{10} \mathrm{Be},{ }^{41} \mathrm{Ca}\right.$, ${ }^{53} \mathrm{Mn}$ ), or stable negative ions of isobars are present $\left({ }^{36} \mathrm{Cl}-{ }^{36} \mathrm{~S},{ }^{59} \mathrm{Ni}-{ }^{59} \mathrm{Co}\right.$, etc. $)$. This method uses the cross-section difference of the recharging process for multicharged ions on gases, e.g., $\mathrm{H}_{2}$ or He. Our calculations show that the $\mathrm{Be} 3+-\mathrm{Be}+$ cross-section is ten times less than $\mathrm{B}^{3+}-\mathrm{B}^{+}$. Thus, about $10 \%$ of $\mathrm{Be}$ ions in the $3+$ state would pass the gas chamber, in contrast to about $10^{-6}$ of $\mathrm{B}^{3+}$ and the selectivity of the step would reach $10^{5}$.

For the ${ }^{36} \mathrm{Cl}-{ }^{36} \mathrm{~S}$ isobar pair selection, the negative ion detachment can be used efficiently (Berkovits et al. 1990). The pulsed ion beam makes it possible to use the time coincidence of laser and ion pulses, and thus, to overcome the main problem of using this technique: low efficiency of laser pulses interaction with a continuous ion beam.

The Trace spectrometer is free from the process producing the ions with the same mass/charge ratio by recharging, which are possible in AMS, for example,

$$
\begin{gathered}
{ }^{12} \mathrm{C}_{3}^{-} \rightarrow{ }^{12} \mathrm{C}^{+} \\
{ }^{36} \mathrm{Cl}^{-} \rightarrow{ }^{36} \mathrm{Cl}^{3+}
\end{gathered}
$$

where the molecule ${ }^{12} \mathrm{C}_{3}^{-}$can be a background for ${ }^{36} \mathrm{Cl}$. Such a process is impossible in Trace due to the reversal recharging method (from positive to negative).

A mass spectrometer of any type (TOF, magnetic or combined) can be used in the next step. It has 


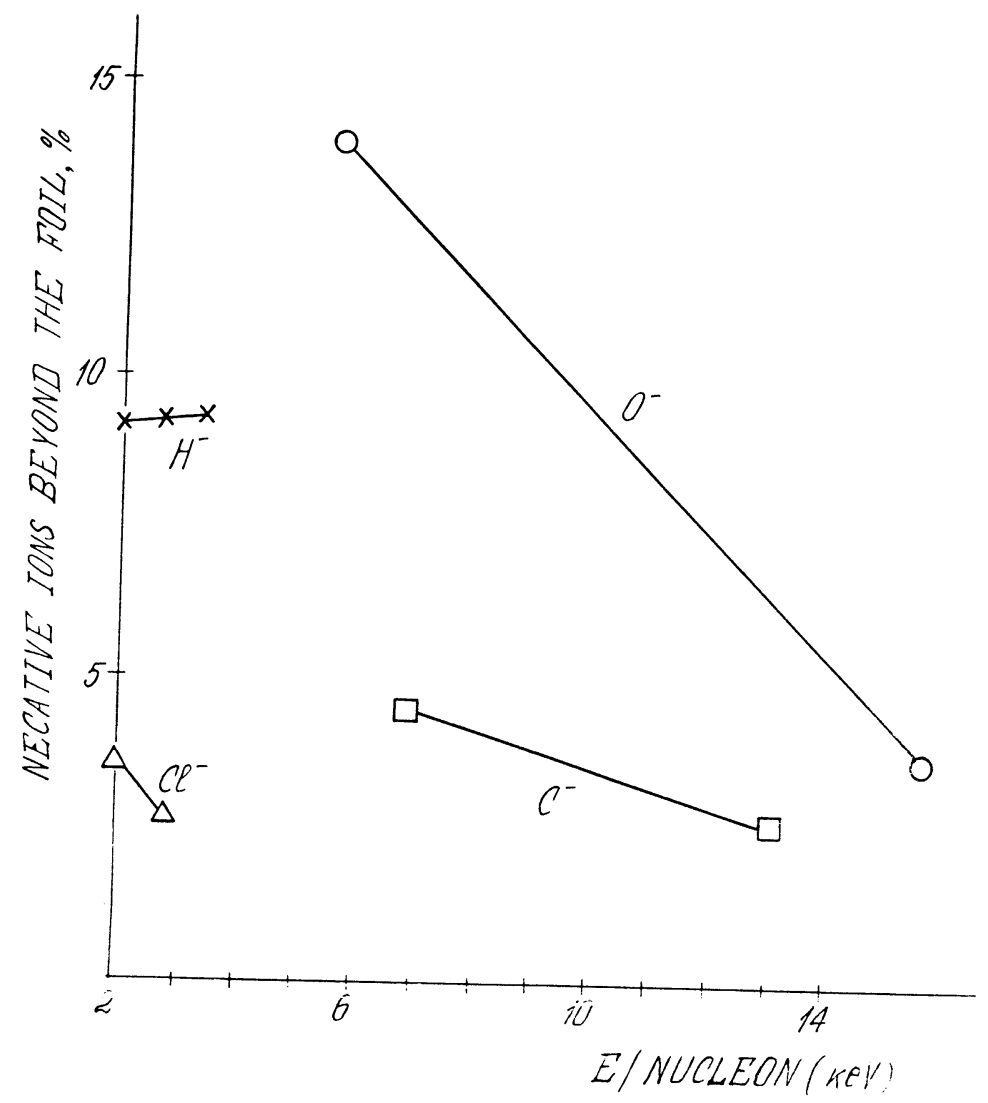

Fig. 5. Production of negative ions beyond the foil

to operate in an ion-energy range of some tens of $\mathrm{keV}$, instead of an MeV range for AMS second mass spectrometers. This peculiarity is advantageous for heavy and very heavy nuclide analysis.

We have also designed another mass-spectrometric scheme based on TOF techniques only (Fig. 6). It is based on a Tandem TOF mass spectrometer with spherical reflectors. This reflector shape allows us to obtain very high sensitivity as a consequence of ion collection from the solid-angle spread to a focus point. Mass resolution is equal to 125 in the first unit and 20 in the second unit, for an energy window of 20-160 eV. Construction of both schemes is now in progress.

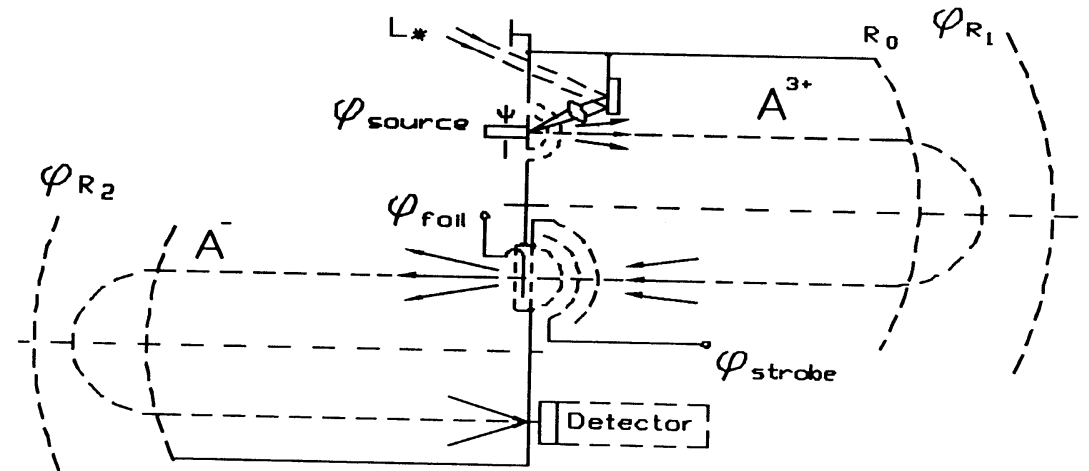

Fig. 6. Time-of-flight mass spectrometer with the spherical mirror 
The opportunity of TOF operating control on all spectrometer units allows us to reduce the background level - the random noise component and the component produced by the scattered ions. The Trace spectrometer can also be used as an effective injector for any type of accelerator, as its ion beam has a pulse form and low intensity $\left(10^{3}-10^{5}\right)$ ions/s.

\section{REFERENCES}

Berkovits, D., Boaretto, E., Hollos, G. Kutschera, W. Naaman, R., Paul, M. and Vager, Z. 1990 Study of laser interaction with negative ions. In Yiou, F. and Raisbeck, G. M., eds., Proceedings of the 5th International Conference on Accelerator Mass Spectrometry. Nuclear Instruments and Methods B52: 384-383.

Kocharov, G., Kogan, V., Konstantinov, A. and Pavlov, A. 1990 The possibilities of cosmogenic isotopes investigation by means of mass-spectrometrical methods. In Yiou, F. and Raisbeck, G. M., eds., Proceedings of the 5th International Conference on Accelerator Mass Spectrometry. Nuclear Instruments and Methods B52: 384-386.

Shima, K. Mikumo, T. and Tawara, H. 1986 Atomic Data and Nuclear Data Tables 34: 57.

Smikk, D. V. and Dubensky, B. 1984 Mirror of massreflectron. Journal of Technical Physics 54: 912-916 (in Russian). 